\title{
Aggressive Thoracic Hemangioma Leading to Secondary Kyphosis with Paraparesis in the Postpartum Period. A Case Report
}

\author{
ANOUAR BOURGHLI, MD, ${ }^{1}$ SALIM M ABDULJAWAD, MD,${ }^{1}$ LOUIS BOISSIÈRE, MD,${ }^{2}$ HANI KHALIFA, \\ $\mathrm{MD},{ }^{3}$ IBRAHIM OBEID, $\mathrm{MD}^{2}$ \\ ${ }^{I}$ Orthopaedic and Spinal Surgery Department, Kingdom Hospital, Riyadh, Saudi Arabia, ${ }^{2}$ Orthopaedic Spinal Surgery Unit 1, Bordeaux Pellegrin Hospital, \\ Bordeaux, France, ${ }^{3}$ Obstetrics and Gynecology Department, Kingdom Hospital, Riyadh, Saudi Arabia
}

\begin{abstract}
Background: Aggressive vertebral hemangioma is rare, and its management can be very challenging.

Methods: In this report, we present the case of a 30-year-old female with a normal twin pregnancy. She had been complaining of midthoracic pain after the seventh month of pregnancy with no other symptoms. Normal delivery was done under spinal anesthesia, but the patient's back pain worsened after the delivery, and she left the hospital on Day 4 with the onset of an unsteady gait. She was seen in the spinal clinic on Day 14, referred by her gynecologist, with a paraparetic presentation that was rapidly worsening. Urgent magnetic resonance imaging (MRI) was done and revealed aggressive hemangioma of T6 with kyphosis and significant cord compression.

Results: The patient was admitted and was operated the following morning. Posterior decompression with fixation from T4 to T8 and kyphosis correction was done. Samples from the vertebrae were sent to the lab for pathology analysis, and the results confirmed the diagnosis of vertebral hemangioma. She could walk on Day 1 with progressive neurological recovery, but distal junctional kyphosis in the form of a "chance fracture" of T8 occurred at 2 months and was treated conservatively. She showed a satisfactory clinical and radiological result at 2 years with no recurrence.

Conclusions: Literature is sparse on the management of vertebral hemangioma in the context of pregnancy and postpartum, with only case reports, and its presentation as a kyphotic fracture is extremely rare. The current case shows how, in the context of delivery, a benign procedure such as spinal anesthesia should be done with extreme caution, as it may play a role in aggravating an undiagnosed aggressive hemangioma; rapid management after the onset of paraparetic symptoms enables a full neurological recovery, but close monitoring later on with regular MRI is mandatory for detection of any recurrence.
\end{abstract}

Tumor

Keywords: aggressive hemangioma, pregnancy, chance fracture

\section{INTRODUCTION}

Vertebral hemangiomas are benign vascular tumors, more common in the female population, mainly located in the thoracic spine, and representing $10-12 \%$ of spinal tumors. ${ }^{1}$ Usually asymptomatic, and mainly discovered randomly on routine MRIs done for other diseases, they may get symptomatic after becoming aggressive. This presentation is rare and represents less than $1 \%$ of all hemangiomas, ${ }^{2}$ and pregnancy is a well-known factor for such a presentation. ${ }^{3,4}$ In fact, hemodynamic and endocrinal changes during the third trimester play a key role in transforming a benign hemangioma to an aggressive one. ${ }^{5}$ Mid or upper back pain represents the main symptom, and luckily, most of the patients will have natural progression toward pain attenuation after delivery, but in some cases, presentation may be more severe, consisting of leg paresthesia, paraparesis, or paraplegia, attesting spinal cord compression because the vertebrae fractured or in case of epidural extension. ${ }^{6}$ These severe symptoms may occur during the third trimester of pregnancy, and in such a case, the treatment may be very challenging when we consider the hazards related to the mother and to the baby, requiring a multidisciplinary approach ${ }^{7}$; or they may occur after delivery, ${ }^{8-11}$ and depending on the presented signs, surgical treatment may become urgent, as any delay in the management may have a direct impact on the neurological recovery later on. 

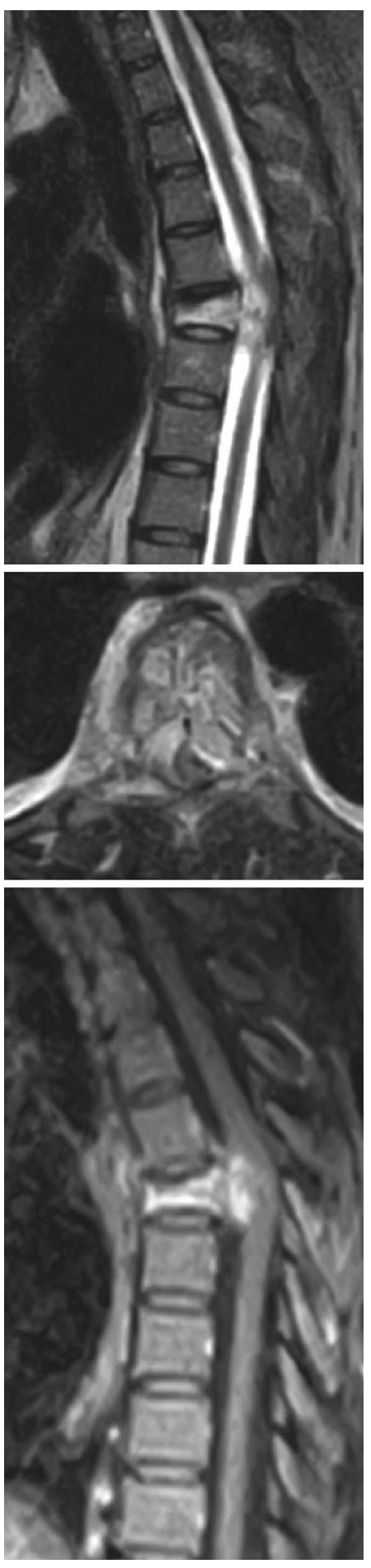

\section{CASE DESCRIPTION}

A 30-year-old female with a normal twin pregnancy had been complaining of midthoracic back pain after the seventh month. Her symptoms were explained by the pregnancy and high weight gain. After reaching the due date, spinal anesthesia was decided for a cesarean section, and she was asked to bend her back as much as possible during the procedure, which increased significantly her existing midback pain. Delivery occurred, and the patient started to walk the following day. She was discharged on Day 4, but with a persisting back pain and the onset of an unsteady gait that was explained as an eventual side effect from spinal anesthesia. Her condition worsened progressively at home, and she came to see her gynecologist on Day 14, which referred her to the spine clinic. Clinical presentation revealed a paraparesis with the patient unable to stand up or walk on her own, with a global lower extremities weakness of $2 / 5$, altered sensation below the breasts (T5-T6 level), enhanced lower extremity reflexes, positive ankle clonus and Babinski bilaterally, and bowel and bladder dysfunction. Urgent magnetic resonance imaging (MRI) was done and revealed a pathological T6 vertebra fracture with kyphosis and significant epidural compression. The vertebra appeared as hypointense on T1 and hyperintense on T2 weighted images, with marked contrast enhancement (Figure 1 (top, middle, and bottom)). Computed tomography (CT) scan confirmed the compression fracture associated to kyphosis, with a coarse trabecular pattern (dense spots within a hypodense lesion), usually characteristic of hemangiomas (Figure 2 (top and bottom)). Given the presentation with progressive paraparesis worsening and cord compression, the patient was operated the following morning.

\section{SURGICAL TECHNIQUE}

The patient was installed in a prone position on 4 cushions. A posterior cutaneous midline incision was made. The spine was exposed subperiosteally from $\mathrm{T} 4$ to $\mathrm{T} 8$ in a fashion similar to other posterior instrumented surgeries, going laterally to the transverse processes. Care was taken to preserve the

Figure 1. MRI showing (top) the T6 compression fracture with hyperintensity signal on T2 weighted images and cord compression, (middle) epidural extension, and (bottom) significant enhancement on T1 with contrast weighted images. 


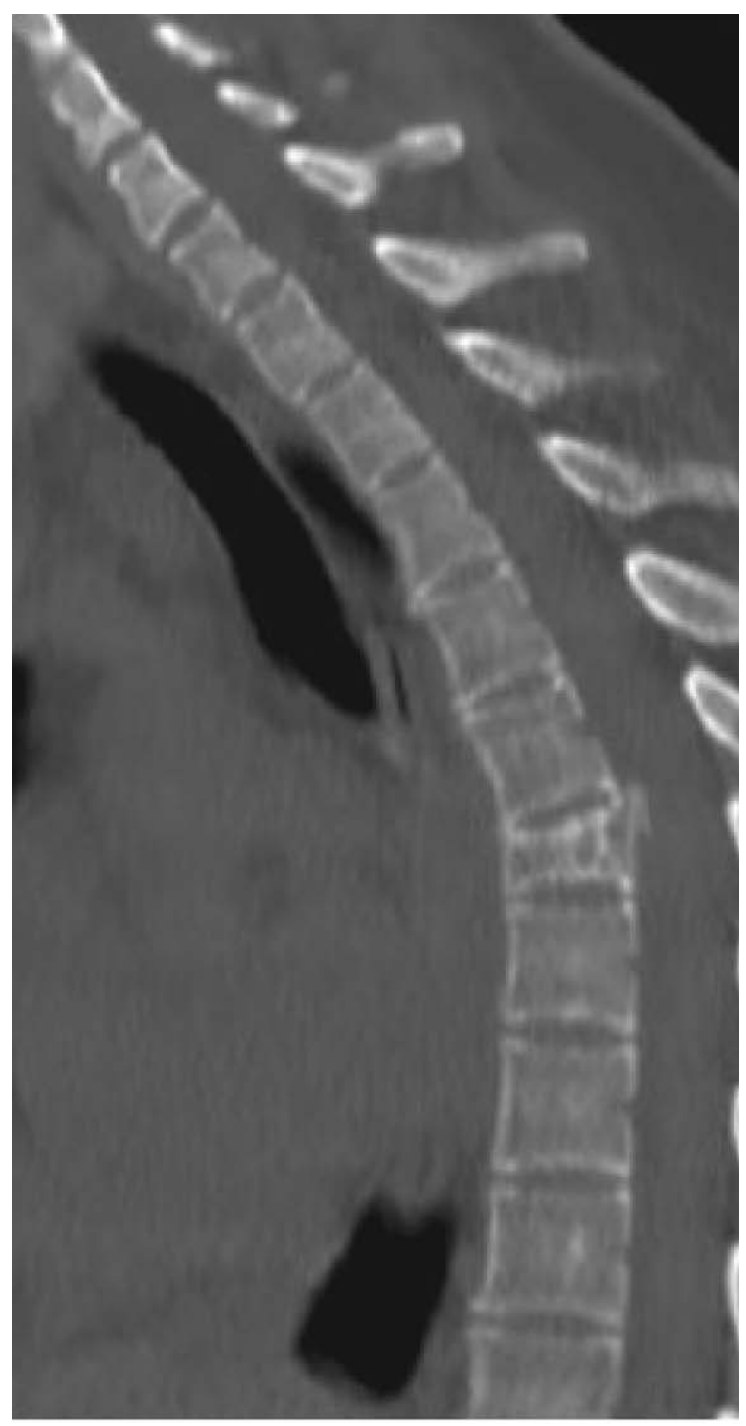

supraspinous and interspinous ligaments between the upper instrumented vertebrae and the level above. Resection of the inferior articular processes at all levels was performed bilaterally to provide maximum flexibility to the spine. The spinous processes were also resected, and the bone recovered prepared for use as a graft at the end of the procedure. We next used the free-hand technique to place the pedicle screws at all levels from T4 to T8 except T6. Screw insertion was difficult in this case due to small thoracic pedicles. T5 and T6 laminectomies were performed. Very turgescent epidural veins could be seen on both sides of the dural sac (Figure 3). Important bleeding was coming from the right-side veins and was controlled with Gelfoam and Surgicel. The left T6 pedicle was entered for the biopsy, and abundant blood came from inside the body, confirming macroscopically a vascular tumor. Bone wax was applied after samples were taken and sent to pathology.

For the correction technique, cantilevering of the spine with 1 straight titanium alloy rod on each side was performed simultaneously; after the rods were blocked distally on $\mathrm{T} 7$ and $\mathrm{T} 8$, reduction towers were put on the proximal screws, pulling the upper thoracic spine to the rods. This was followed by the final tightening of the setscrews proximally, and the prepared grafts were placed to cover the maximum surface. Patient required blood transfusion (2 units), as total blood loss was $0.8 \mathrm{~L}$.

Postoperatively, the course was uncomplicated. The patient was authorized to walk the first day postsurgery, and a single drain was removed on Day 3.

\section{RESULTS}

The patient could walk on Day 1 with a walker and the assistance from a physical therapist. Postoperative CT scan confirmed correction of the kyphosis with good screw positioning (Figure 4). Standing x-rays were done on Day 4, showing a good sagittal and coronal alignment on both views (Figure 5 (left and right)). Samples taken from the T6 vertebra confirmed the diagnosis of hemangioma (cavernous type). Gradual neurological recovery occurred. She replaced the walker with a single cane at 3 weeks and stopped using the latter at 6 weeks.

Figure 2. CT scan confirming (top) the compression fracture with kyphotic angulation and (bottom) a coarse trabecular pattern typical of vertebral hemangioma. 


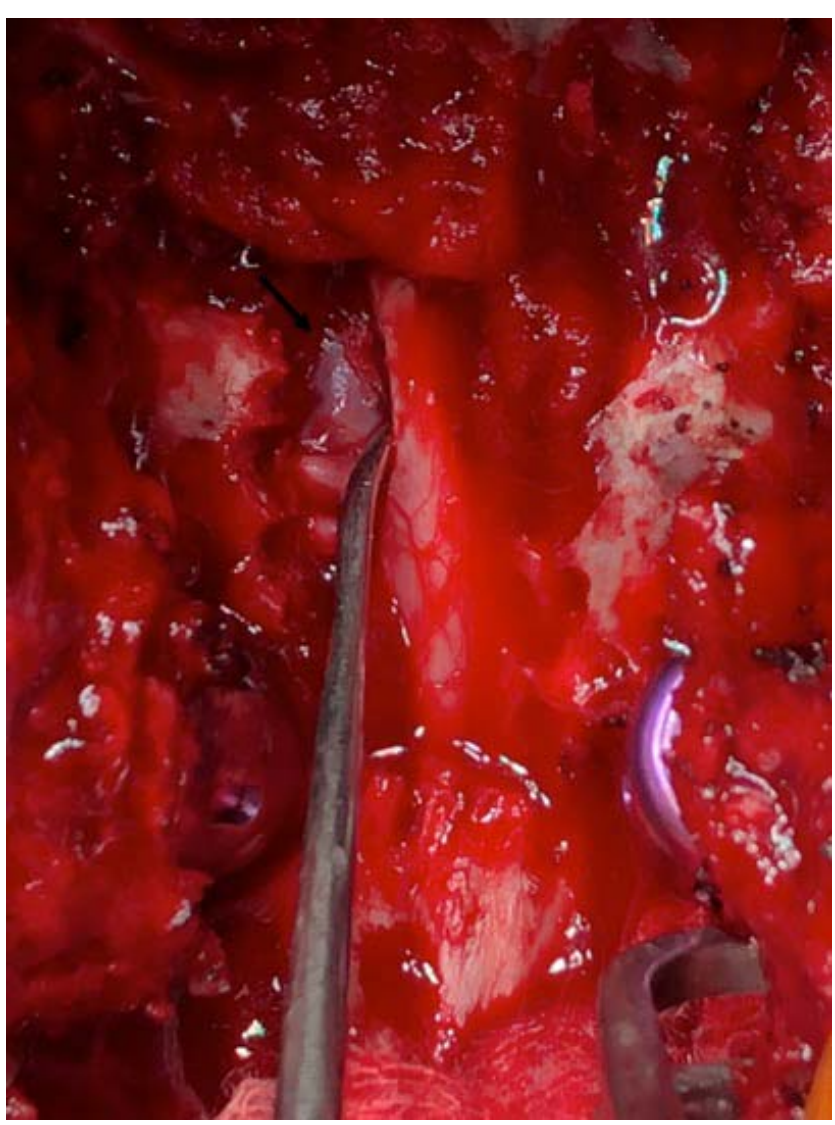

Figure 3. Intraoperative image showing turgescent epidural veins (black arrow).

She complained of an increasing midback pain at 2 months, different from the initial one, and x-ray revealed distal junctional kyphosis. CT scan showed "chance fracture" of T8 (Figure 6 (left and center)), leading to a local kyphosis T8-T9 (Figure 6 (right)). Patient was treated conservatively with a brace for 2 months, as she refused any surgical management, and follow up did not show any worsening of the local kyphosis.

She returned to her job as a schoolteacher at 3 months, with remaining paresthesia on the left side of her chest (T6 dermatome). MRI done at 6 months showed satisfactory cord decompression with no recurrence (Figure 7 (top, middle, and bottom)).

She showed satisfactory clinical and radiological results at 2 years (Figure 8 (left and right)).

\section{DISCUSSION}

The first case of symptomatic vertebral hemangioma related to pregnancy was described by Guthkelch in $1948,{ }^{12}$ and since then, more than 30 cases

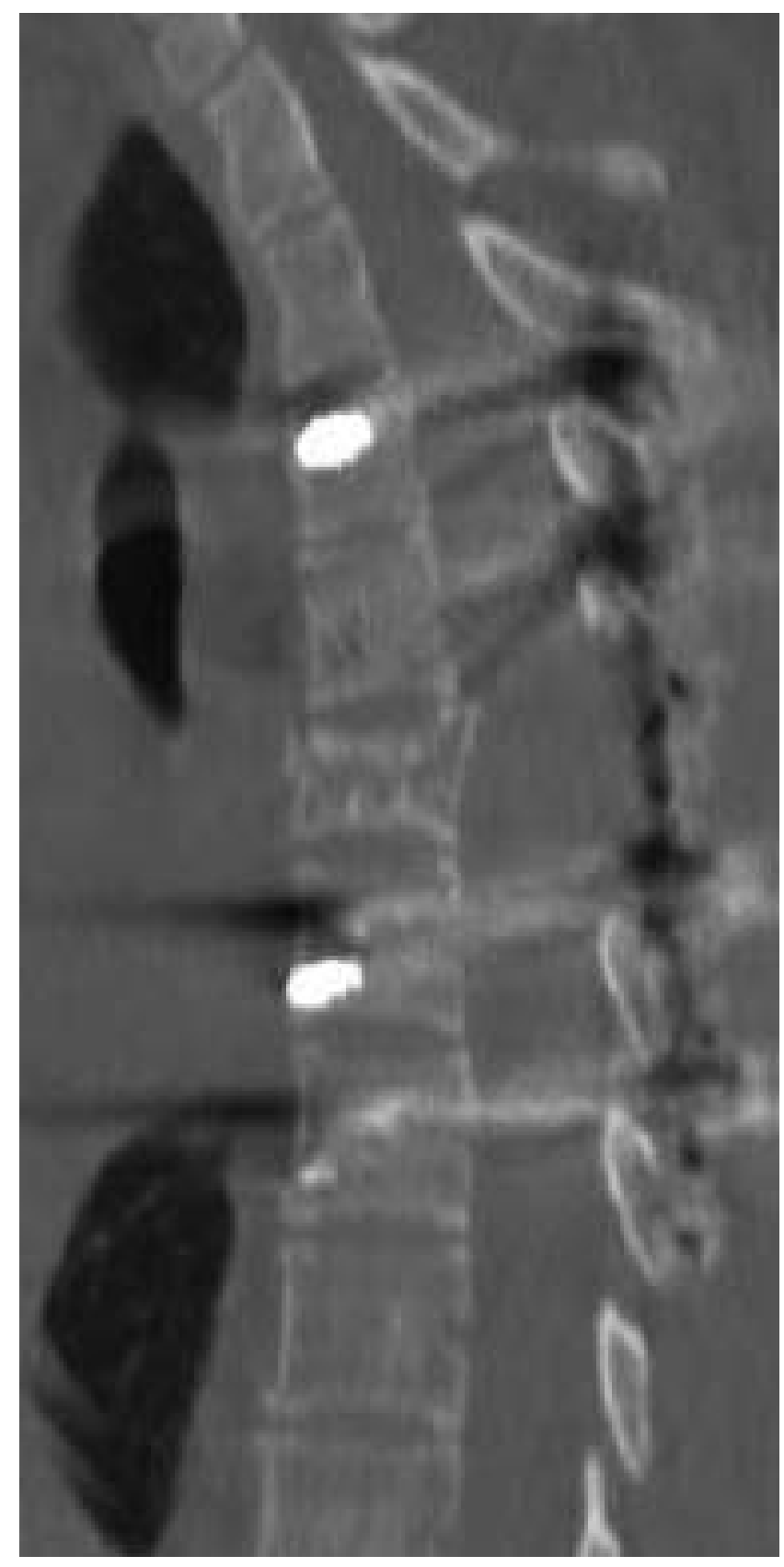

Figure 4. Postoperative CT scan confirming good correction of the kyphosis.

have been reported in the literature, mostly during the third trimester, with a single case in the second trimester, but in the postpartum period, only 4 cases could be retrieved from the literature. ${ }^{8-11}$

The hemodynamic changes that affect pregnant women peak in the third trimester, and the pathophysiological approach to understand it is that the gravid uterus causes venous obstructions and increases the intra-abdominal pressure, which leads to redistribution of blood flow, maximizing it through the vertebral venous plexus, which leads to 


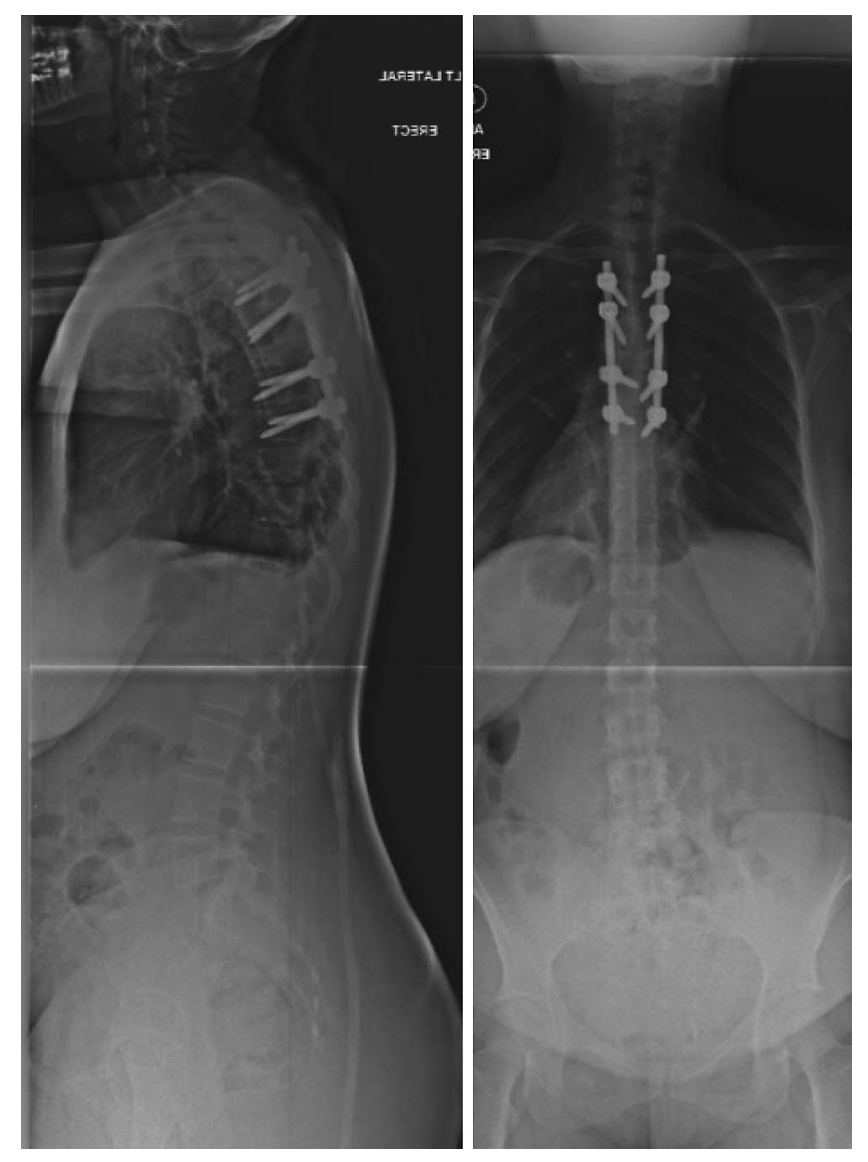

Figure 5. (left) and (right) Standing x-rays done on D4 after the surgery showing a good global alignment on both views. expansion and enlargement of an asymptomatic vertebral hemangioma. ${ }^{13,14}$ Also, the high levels of progesterone and estrogen in the third trimester increase the endothelial growth and the venous distensibility due to the increase in plasma volume, which is an additional factor that contributes to the formation and transformation of a vertebral hemangioma. $^{15}$

Most of the reported cases were in the thoracic spine with few described cases in the lumbar ${ }^{11}$ and cervical region. The most common presenting symptom is back pain, followed by paraparesis or paraplegia; cord compression can be caused by 1 of the following reasons: epidural mass causing nerves compression due to subperiosteal expansion of the hemangioma, acute hemorrhage to the epidural space, spinal cord ischemia, hypertrophy of the posterior cortex of the vertebral body, and infrequently compression fracture of the involved vertebral body. ${ }^{1,14}$ Compression fracture is actually a rare mechanism because the involved vertebra has usually dense trabeculae that protect it from axial collapse. On plane radiographs, this pattern of dense trabeculae is described as "jail bar" appearance, and on axial CT scan, it is characteristically seen as dense spots within a hypodense lesion referred to as "polka dot" pattern. ${ }^{16}$ However,
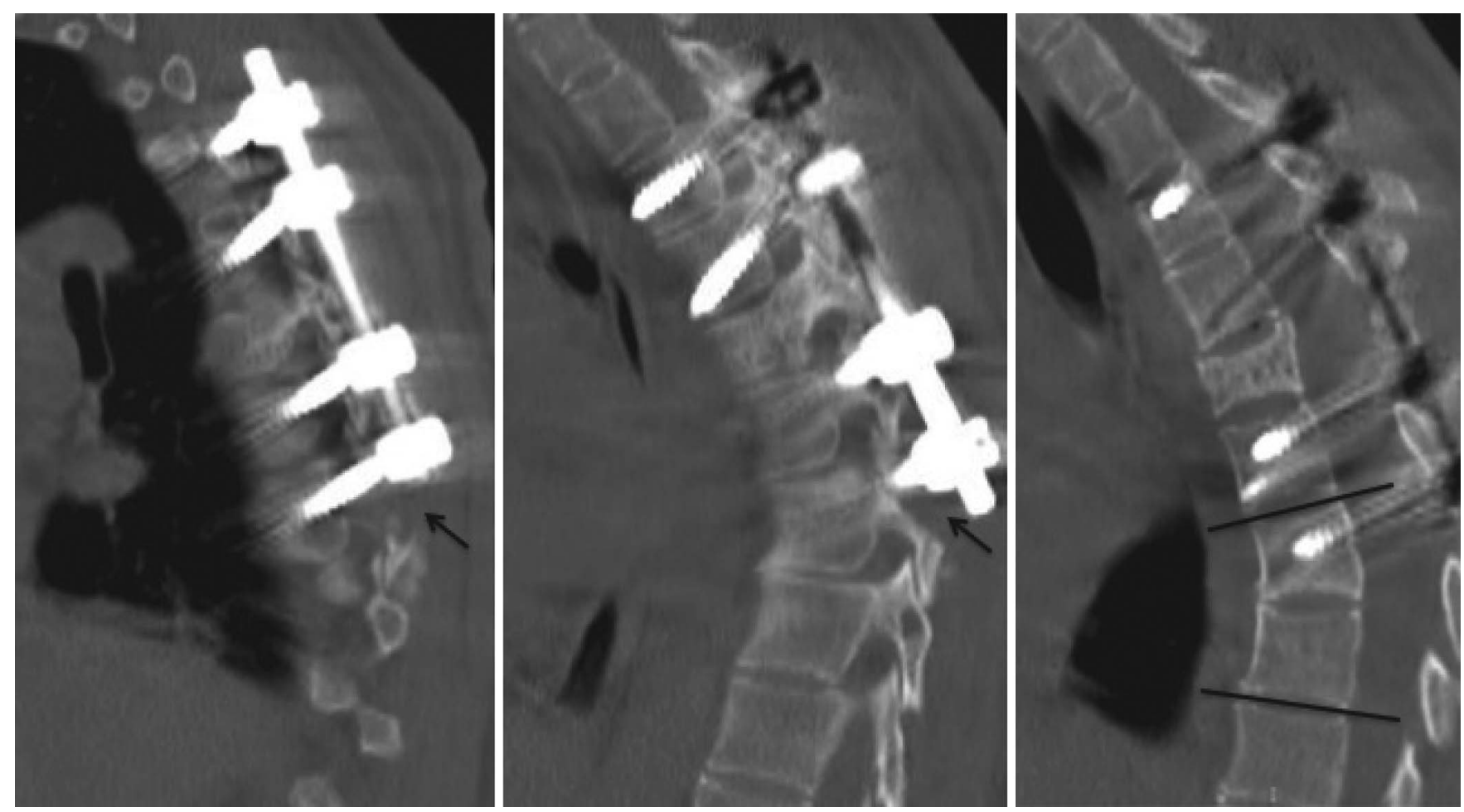

Figure 6. CT scan at 2 months showing (left) and (center) distal junctional kyphosis in the form of a "chance fracture" of T8 (black arrows), leading to (right) a local kyphosis T8-T9 (black lines). 

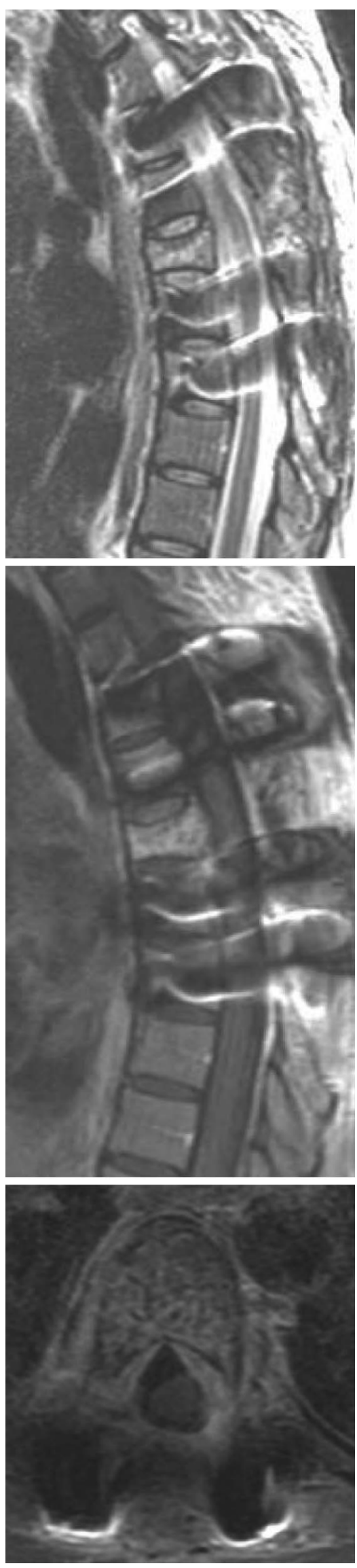

Figure 7. MRI done at 6 months showing (top, middle, and bottom) satisfactory cord decompression with no recurrence.

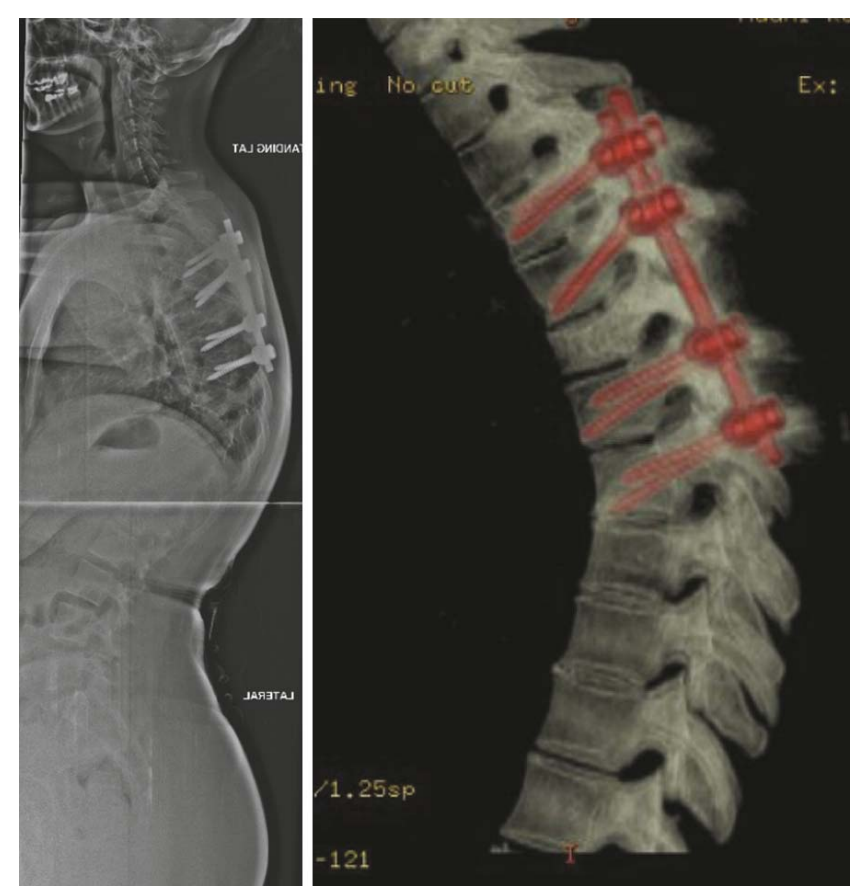

Figure 8. Full spine $x$-ray at 2 years confirming (left) the stable status with good alignment and (right) the healed distal chance fracture of T8 on CT scan.

MRI remains the best diagnostic modality for the diagnosis of vertebral hemangioma, especially during pregnancy (to avoid any radiation), but also after delivery, as it shows a typical aspect with hyperintensity on $\mathrm{T} 1$ and $\mathrm{T} 2$ weighted images due to more fat components and less vascular stroma in normal hemangioma. However, aggressive hemangioma becomes hypo to isointense on $\mathrm{T} 1$ and hyperintense on $\mathrm{T} 2$ weighted images due to high vascularity and less fat. ${ }^{17}$

There are 2 types of vertebral hemangiomas according to the histological appearance: cavernous and capillary. The most common type is the cavernous one, as in our case, which is characterized by large sinusoidal spaces lined by a single layer of epithelium, while the capillary type has smaller vascular channels. ${ }^{5}$

Treatment of hemangioma is indicated either when the patient develops severe pain or if neurological impairment occurs. Management in the postpartum period may offer different options such as surgical decompression, radical resection, vertebroplasty, radiation therapy, and embolization. ${ }^{18}$ The last 2 options are formally contraindicated during pregnancy.

However, there is a controversy in the surgical management of paraparesis after posthemangioma pathological fracture - no evidence-based guidelines 
Aggressive Thoracic Hemangioma Leading to Secondary Kyphosis with Paraparesis in the Postpartum Period

Table. Cases of symptomatic vertebral hemangiomas in the postpartum period reported in the literature.

\begin{tabular}{lccclc}
\hline Authors and Year & Maternal Age & Level & Clinical Features & Treatment & Recovery \\
\hline Schwartz et al 2000 & 29 & T11 & Paraparesis & Anterior partial corpectomy and fusion, radiotherapy & Complete \\
Jain et al 2014 & 26 & T9 & Paraparesis & Patient refused surgery, lost to follow up & NA \\
Staikou et al 2015 & 32 & L2 & Paraparesis & Laminectomy, posterior fusion & Complete \\
Mohanty et al 2018 & 29 & T2 & Paraparesis & Patient refused surgery, intravenous steroids & Complete \\
Present study & 30 & T6 & Paraparesis & Laminectomy, kyphosis correction, fusion & Complete \\
\hline
\end{tabular}

Abbreviation: NA, not available.

exist because mainly case reports are found in the literature. The main recommendation is to proceed with a posterior decompression without tumor resection, considering its benign nature and the fact that neurological recovery will be observed in most of the patients. Some reports described radical resection by spondylectomy in the case of ventrally located lesions with bony compression or in the case of recurrence of a previously operated hemangioma. ${ }^{7}$ Nevertheless, spondylectomy should be very specifically indicated, as it is a technically demanding procedure, and it can cause substantially great intraoperative blood loss. ${ }^{10,19,20}$

Only 4 cases of postpartum aggressive hemangioma, similar to the current case, were found in the literature, and in 3 of the 4 cases, the neurological symptoms started immediately after the delivery. Their presentation and management are summarized in the Table.

In the case of our patient, given the progressive onset of paraparesis in the postpartum period with secondary kyphosis, she underwent posterior decompression with kyphosis correction and fixation, which enabled full motor recovery. It was nevertheless complicated by an uncommon distal junctional kyphosis in the form of a chance fracture that was treated conservatively, with a satisfactory clinical recovery with no recurrence at 2 years. Retrospectively and given the history of facts, her vertebra probably fractured during or immediately after the spinal anesthesia when she was asked to bend forward as much as possible. This should raise a red flag when performing such a procedure if a pregnant woman has been complaining of midback pain in the past several months without an obvious diagnosis or in case a vertebral hemangioma has been already diagnosed.

\section{REFERENCES}

1. Fox MW, Onofrio BM. The natural history and management of symptomatic and asymptomatic vertebral hemangiomas. J Neurosurg. 1993;78(1):36-45. doi:10.3171/jns. 1993.78.1.0036.
2. Chi JH, Manley GT, Chou D. Pregnancy-related vertebral hemangioma. Case report, review of the literature, and management algorithm. Neurosurg Focus. 2005;19(3):E7.

3. Gupta M, Nayak R, Singh H, Khwaja G, Chowdhury D. Pregnancy related symptomatic vertebral hemangioma. Ann Indian Acad Neurol. 2014;17(1):120-122. doi:10.4103/09722327.128577.

4. Moles A, Hamel O, Perret C, Bord E, Robert R, Buffenoir K. Symptomatic vertebral hemangiomas during pregnancy. $J$ Neurosurg Spine. 2014;20(5):585-591. doi:10.3171/2014.2. SPINE13593.

5. Slimani O, Jayi S, Fdili Alaoui F, et al. An aggressive vertebral hemangioma in pregnancy: a case report. $J$ Med Case Rep. 2014;8:207. doi:10.1186/1752-1947-8-207.

6. Tucer B, Ekici MA, Menku A, Koc RK, Guclu B. Surgical management of symptomatic T8 vertebral hemangioma: case report and review of the literature. Turk Neurosurg. 2013;23(5):680-684. doi:10.5137/1019-5149.JTN.6371-12.1.

7. Vijay K, Shetty AP, Rajasekaran S. Symptomatic vertebral hemangioma in pregnancy treated antepartum. A case report with review of literature. Eur Spine J. 2008;17 Suppl 2:S299-S303. doi:10.1007/s00586-008-0592-2.

8. Jain RS, Agrawal R, Srivastava T, Kumar S, Gupta PK, Kookna JC. Aggressive vertebral hemangioma in the postpartum period: an eye-opener. Oxf Med Case Reports. 2014;2014(7):122-124. doi:10.1093/omcr/omu048.

9. Mohanty CR, Jain M, Dash C, Behera SS. Postpartum paraparesis due to aggressive vertebral hemangioma causing spinal cord compression presenting for emergency cesarean section. J Obstet Gynaecol India. 2019;69(2):185-187. doi:10. 1007/s13224-018-1194-5.

10. Schwartz TH, Hibshoosh H, Riedel CJ. Estrogen and progesterone receptor-negative T11 vertebral hemangioma presenting as a postpartum compression fracture: case report and management. Neurosurgery. 2000;46(1):218-221.

11. Staikou C, Stamelos M, Boutas I, Koutoulidis V. Undiagnosed vertebral hemangioma causing a lumbar compression fracture and epidural hematoma in a parturient undergoing vaginal delivery under epidural analgesia: a case report. Can J Anaesth. 2015;62(8):901-906. doi:10.1007/s12630015-0381-4.

12. Guthkelch AN. Haemangiomas involving the spinal epidural space. J Neurol Neurosurg Psychiatry. 1948;11(3):199210. doi:10.1136/jnnp.11.3.199.

13. Nelson DA. Spinal cord compression due to vertebral angiomas during pregnancy. Arch Neurol. 1964;11:408-413.

14. Tekkok IH, Acikgoz B, Saglam S, Onol B. Vertebral hemangioma symptomatic during pregnancy - report of a case and review of the literature. Neurosurgery. 1993;32(2):302-306; discussion 306. doi:10.1227/00006123-199302000-00022. 
15. Mealey J Jr, Carter JE. Spinal cord tumor during pregnancy. Obstet Gynecol. 1968;32(2):204-209.

16. Dang L, Liu C, Yang SM, et al. Aggressive vertebral hemangioma of the thoracic spine without typical radiological appearance. Eur Spine J. 2012;21(10):1994-1999. doi:10.1007/ s00586-012-2349-1.

17. Friedman DP. Symptomatic vertebral hemangiomas: MR findings. AJR Am J Roentgenol. 1996;167(2):359-364. doi:10.2214/ajr.167.2.8686604.

18. Wang B, Jiang L, Wei F, Liu XG, Liu ZJ. Progression of aggressive vertebral hemangiomas during pregnancy: three case reports and literature review. Medicine (Baltimore). 2018;97(40):e12724. doi:10.1097/MD.0000000000012724.

19. Acosta FL Jr, Dowd CF, Chin C, Tihan T, Ames CP, Weinstein PR. Current treatment strategies and outcomes in the management of symptomatic vertebral hemangiomas. Neurosurgery. 2006;58(2):287-295; discussion 287-295. doi:10.1227/ 01.NEU.0000194846.55984.C8.

20. Shinozaki M, Morita A, Kamijo K, Seichi A, Saito N, Kirino T. Symptomatic T2 vertebral hemangioma in a pregnant woman treated by one stage combination surgery; posterior stabilization and anterior subtotal tumor resection. Case report. Neurol Med Chir (Tokyo). 2010;50(8):674-677. doi:10.2176/ nmc.50.674.

Disclosures and COI: There are no conflicts of interest for this case report.

Corresponding Author: Anouar Bourghli, MD, Orthopaedic and Spine Surgery Department, Kingdom Hospital, PO Box 84400, Riyadh 11671, Saudi Arabia. Phone: (+966) 275-0234; Fax: $(+966)$ 275-1111; Email: Anouar.bourghli@gmail.com.

Published 30 June 2020

This manuscript is generously published free of charge by ISASS, the International Society for the Advancement of Spine Surgery. Copyright (c) 2020 ISASS. To see more or order reprints or permissions, see http://ijssurgery.com. 